$$
\begin{aligned}
& \text { واكمُ بميرابير } \\
& \text { انجارنشثب اروو،جامع اروو،كرايق } \\
& \text { واكثم ثميند لياتث }
\end{aligned}
$$

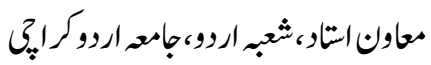

$$
\begin{aligned}
& \text { قै }
\end{aligned}
$$

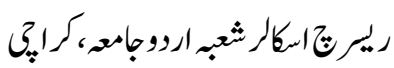

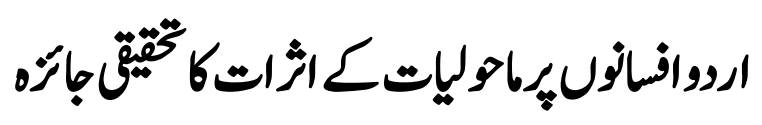

Dr. Sumera Bashir

Incharge Department of Urdu, Urdu University, Karachi.

Dr. Samreena Liaqat

Co-Teacher, Department of Urdu, Urdu University, Karachi

Shama Aqil

Research Scholar, Department of Urdu, Urdu University, Karachi.

\title{
An Analysis of Ecological Effects on the Urdu Short Story
}

The literal meaning of Ecology is surrounding it means all the things that effect the living beings. It is called Ecology.In fact, the study of Ecology comprises of the study of Biology, Mathematics, Geology, Geography, History, Economics, Fine Arts, Geological Science Water study.Through the study of ecology, we become aware of all the characteristics, processes, elements, qualities and weaknesses of our surroundings. As the world makes progress in the field of scientific technology, the industrial advancement and intensity of seasons, water pollution atomic explosions and the depletion of the ozone layer are such activities/processes which have made a negative impact on the ecology. The Earth becomes useless with natural disasters such as famine, earthquakes, floods and heavy rainfalls. These Ecology course has not brought about changes on the earth heavens, atmosphere and rivers in the present times only, but have been active since ancient times. For instance, the people of HazratSalehwere destroyed because of an earthquake, the prophet 
looth's people were destroyed by stones, and the people of HazratNooh were met with destruction by a severe black storm.Ecology changes not only effect the earth, atmosphere or rivers etc., but they make a deep effect on the human behavior.Besides debates, demonstrations and rallies, newspapers and magazines, posters books, media and literature is an effective source of giving environmental awareness.Our story writers have given attention to the effects brought about on human behavior due to the intensity of seasons, famine struck earth and atomic explosions and have made it a theme for their short stories.

Keywords: Short Story, Ecology, Geological Science, Atomic Explosions, Earthquake.

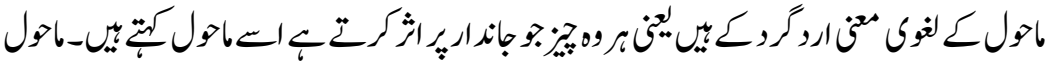

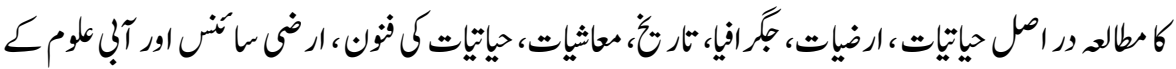

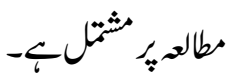

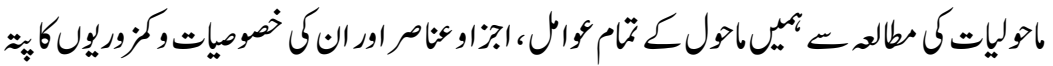

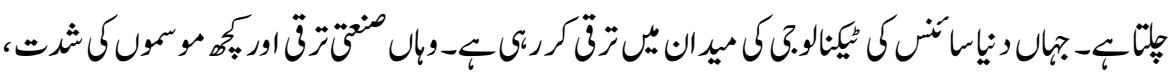

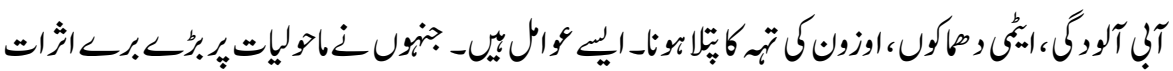

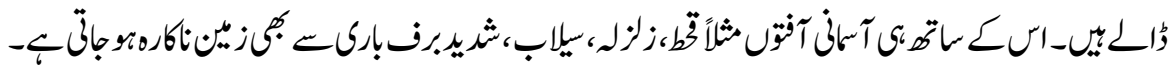

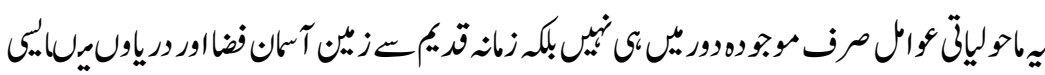

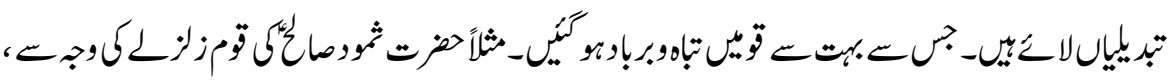

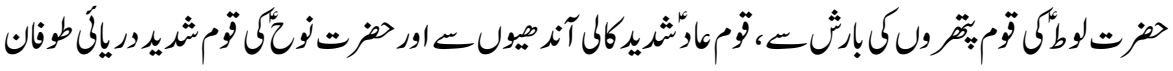

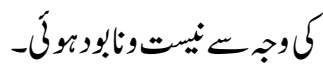

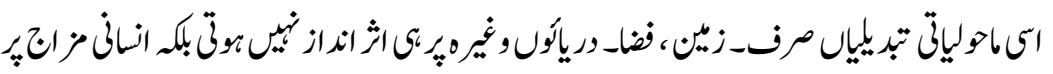

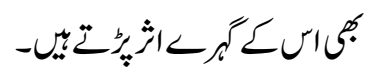

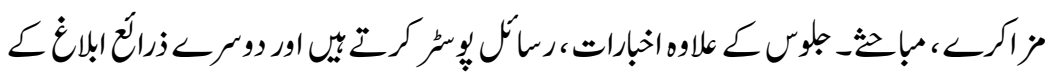

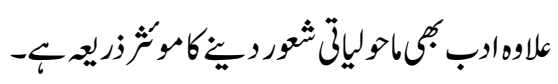


ISSN (Print): 2709-9636 | ISSN(Online) : 2709-9644 | ISSN-L : 2709-9636

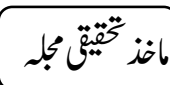

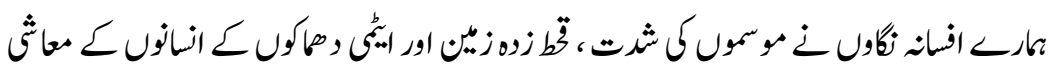

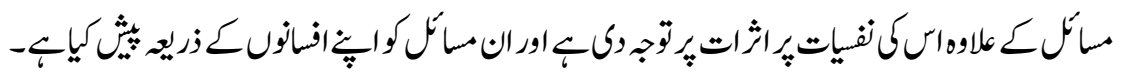

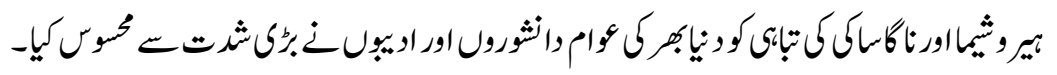

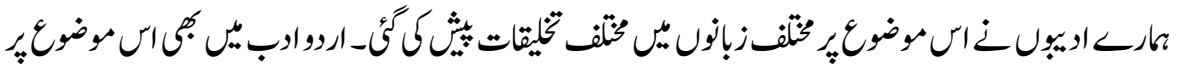

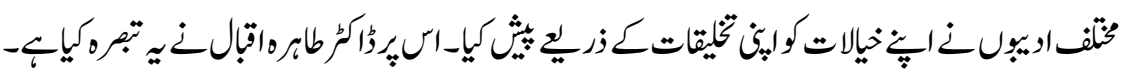

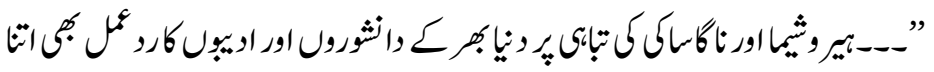

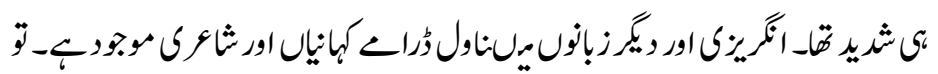

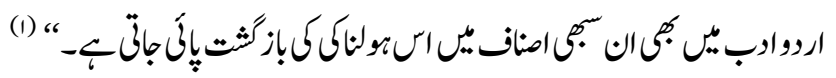

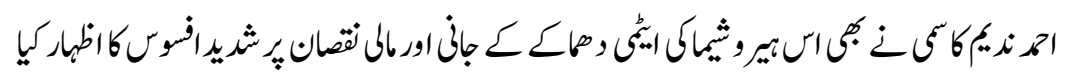

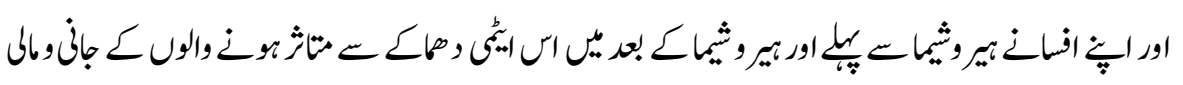

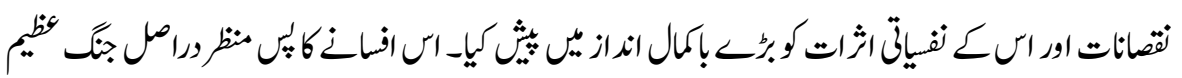

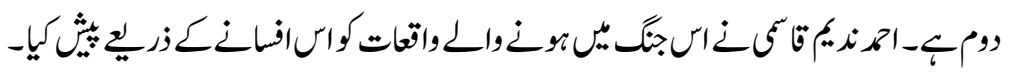

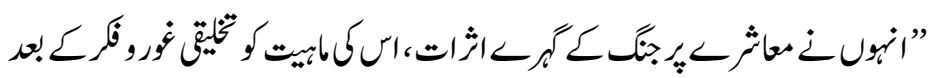

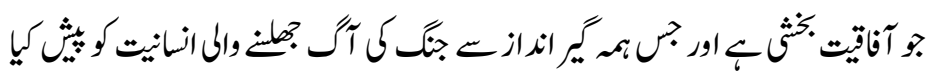

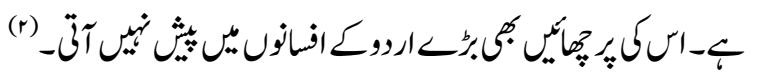

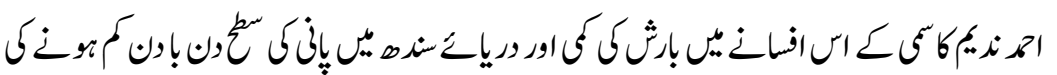

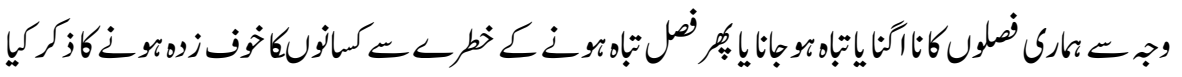

$-4$

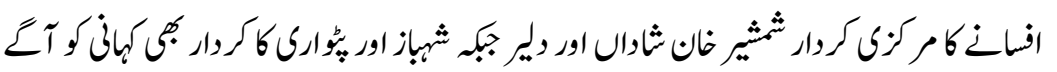

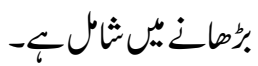

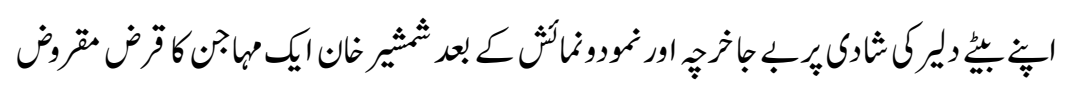

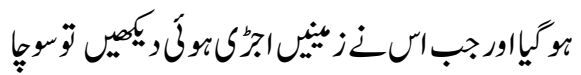

$14 r$ 


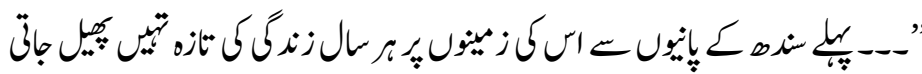

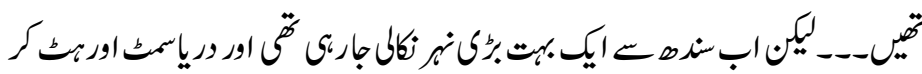

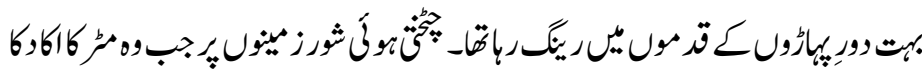

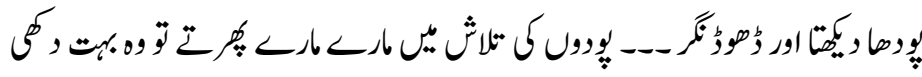
(r) (ب)

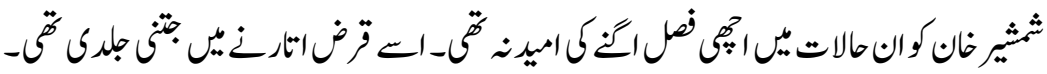

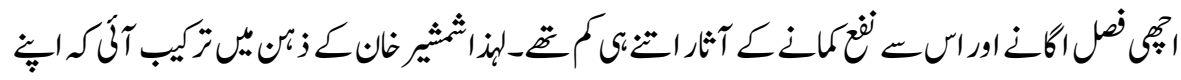

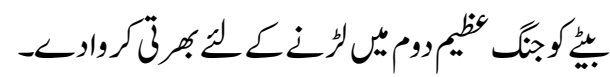

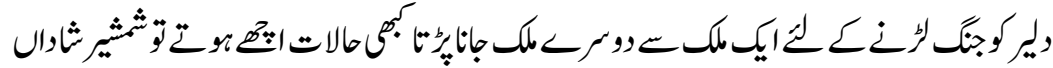

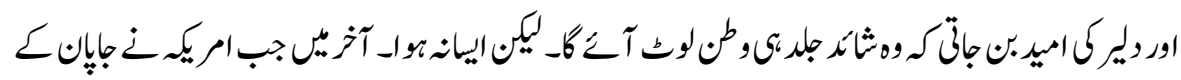

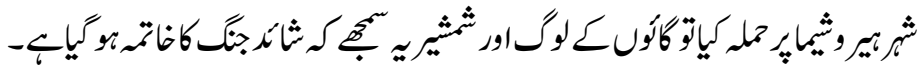

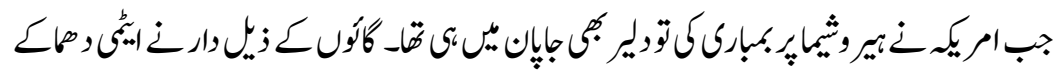
كطاتث عباربين:تيايك

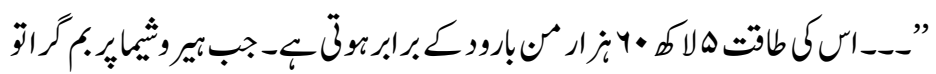

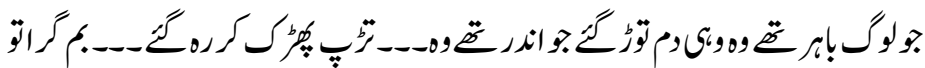

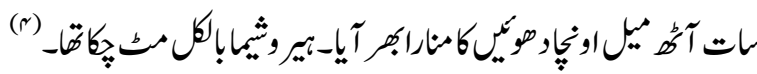

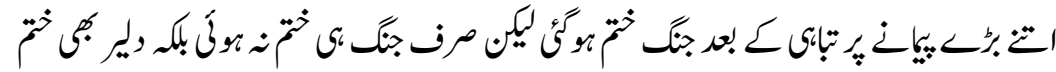

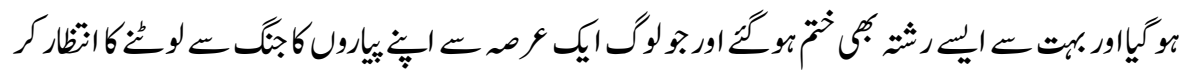

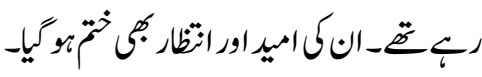

$$
\begin{aligned}
& \text { ع المامكجوع }
\end{aligned}
$$

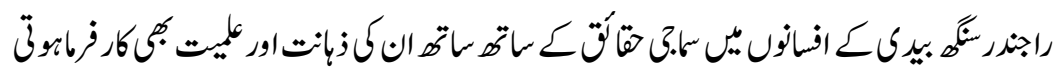

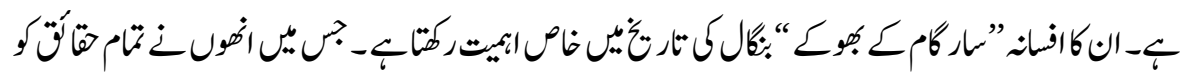

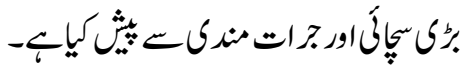


ISSN (Print): 2709-9636 | ISSN(Online) : 2709-9644 | ISSN-L : 2709-9636

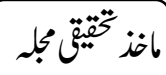

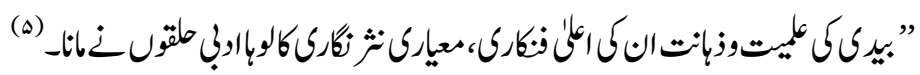

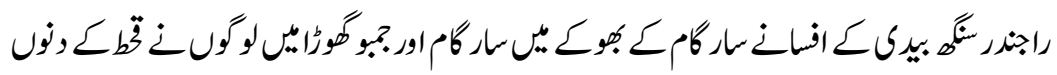

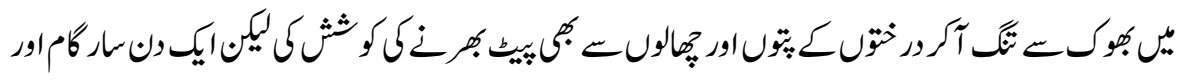

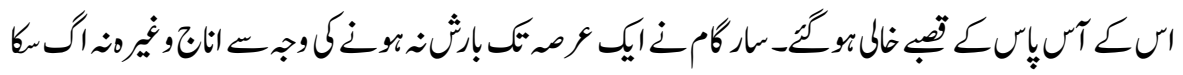

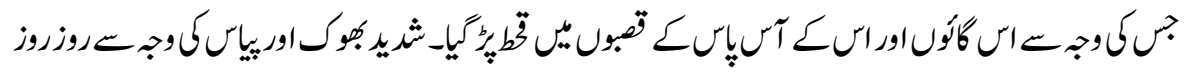

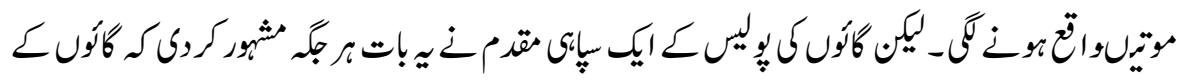

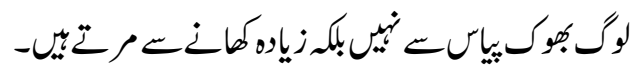

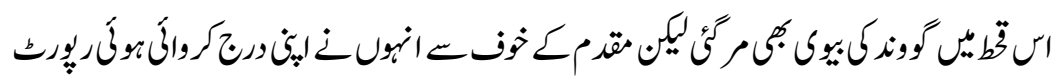

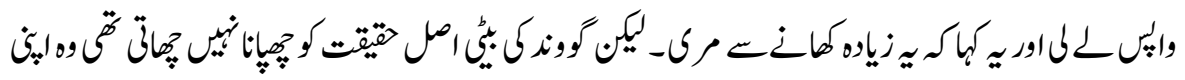

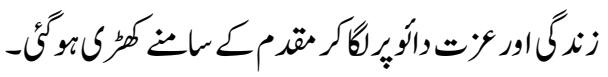

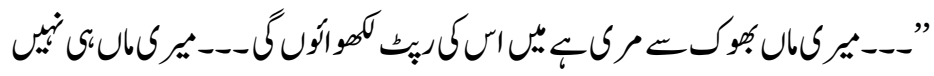

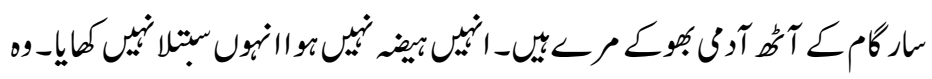

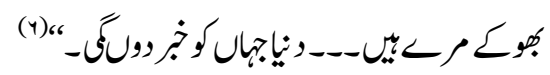

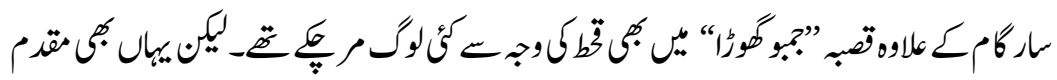

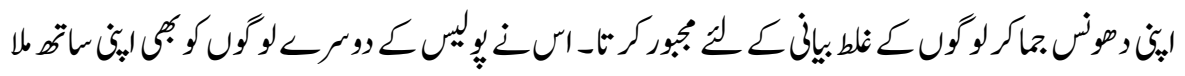

لي

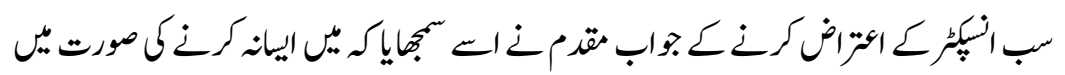

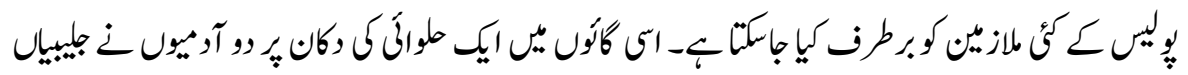

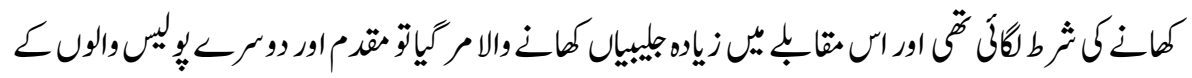

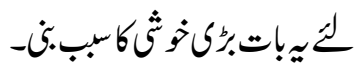

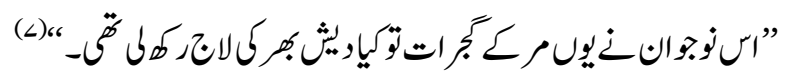

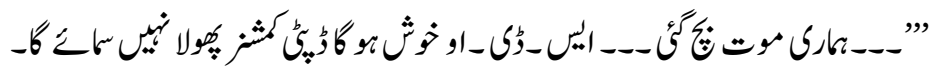

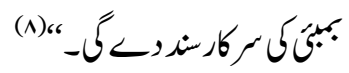

144 


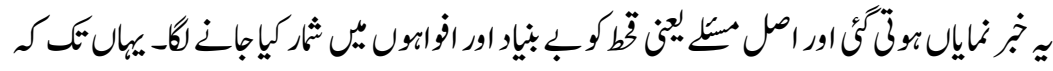

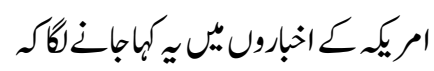

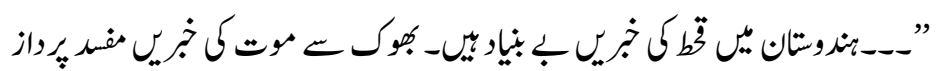

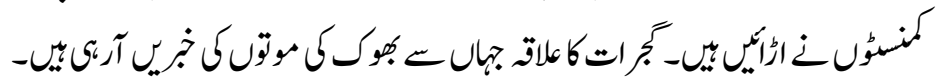

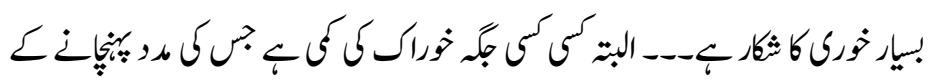

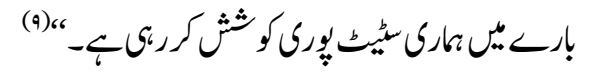

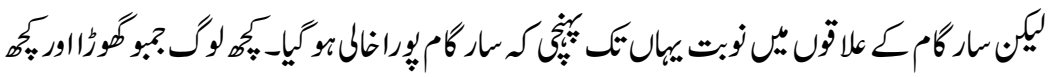

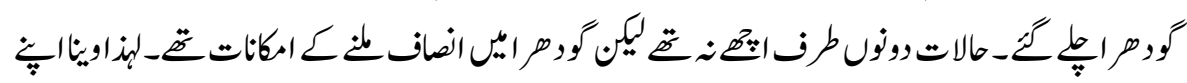

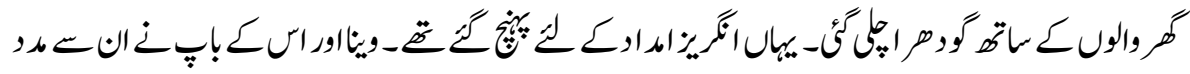

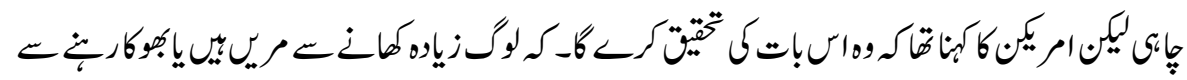

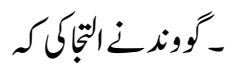

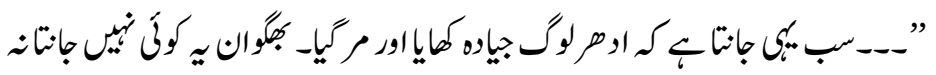

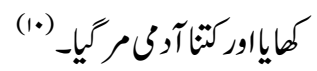

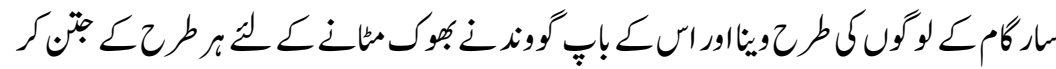

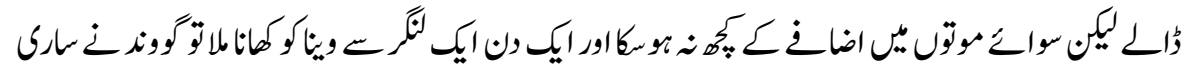

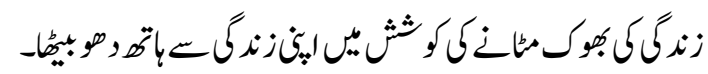

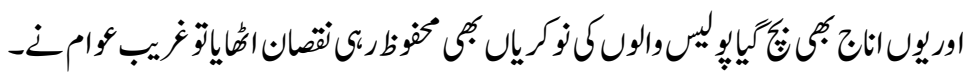




\section{"ֶֶֶטكرات"}

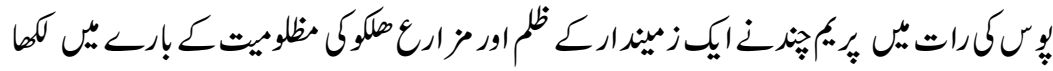

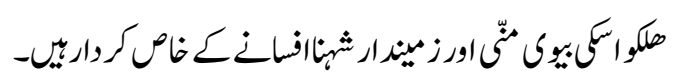

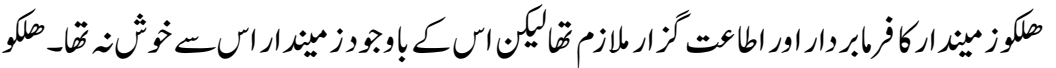

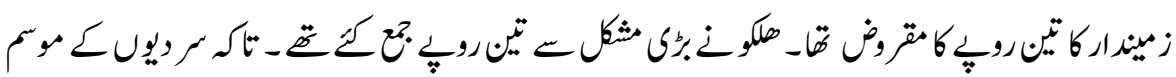

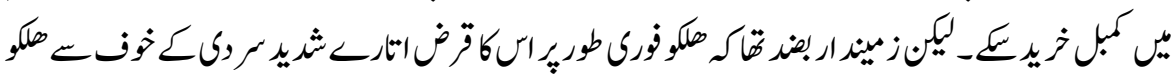

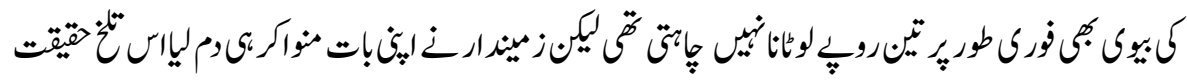

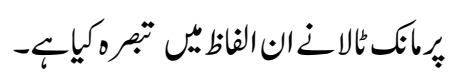

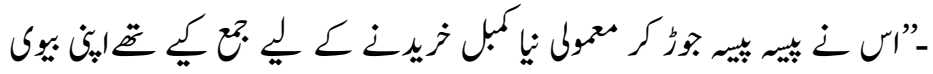

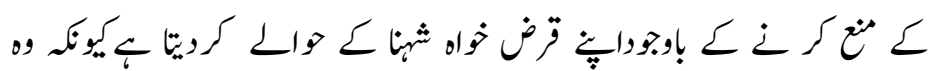

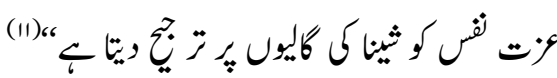

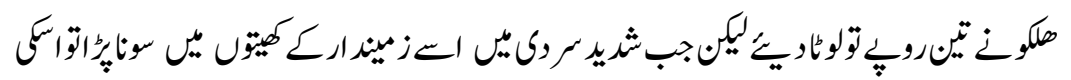

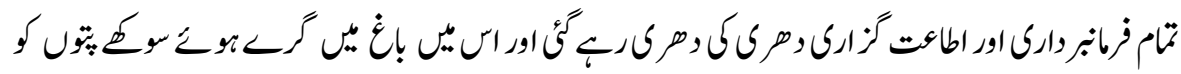

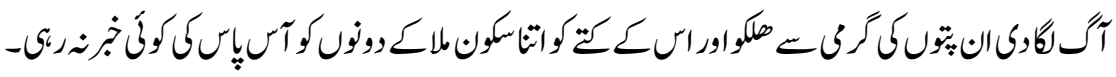

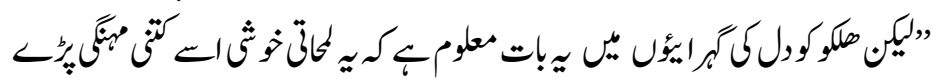

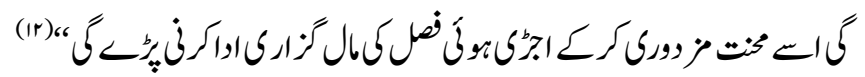

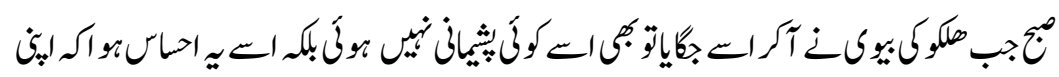

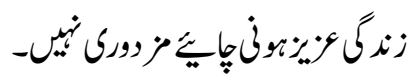




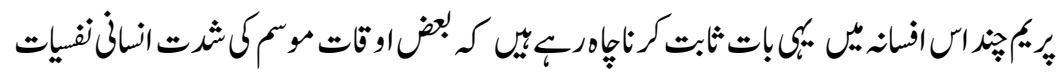

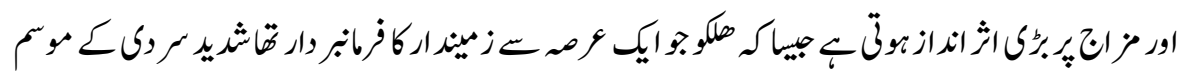

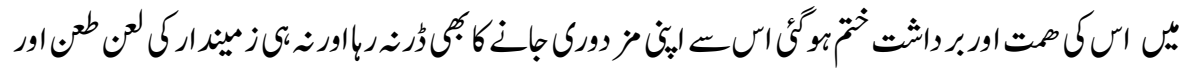

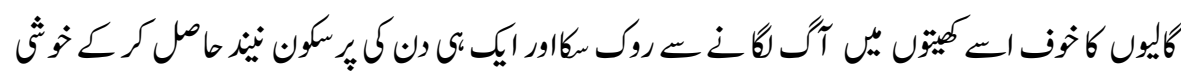

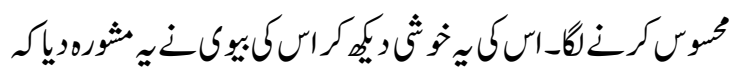

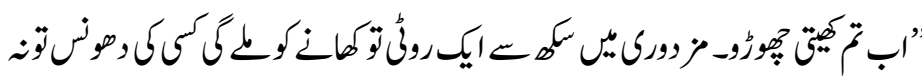

$$
\text { (ir) }-\xi^{2}
$$

"آن رات"

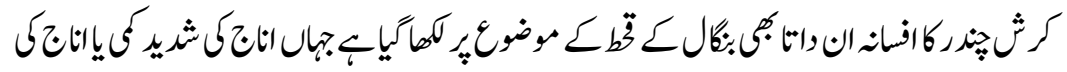

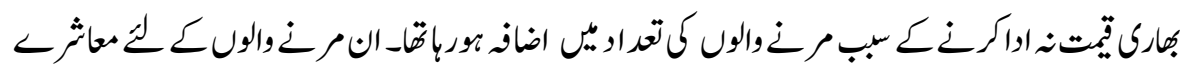

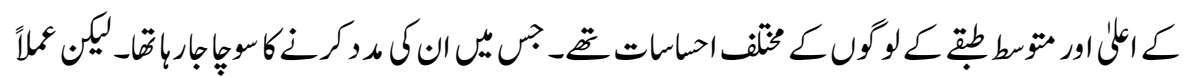

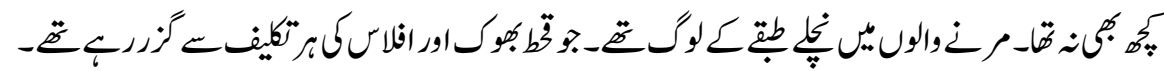

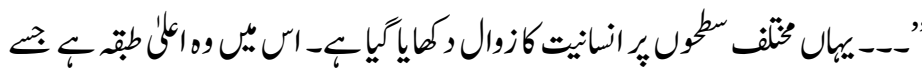

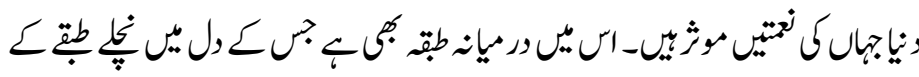

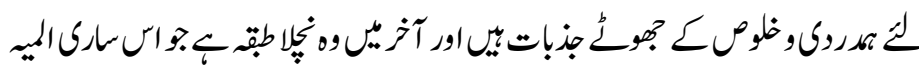

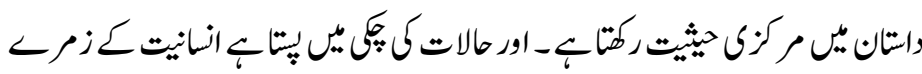
(ir)،

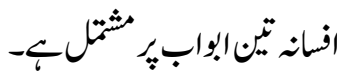

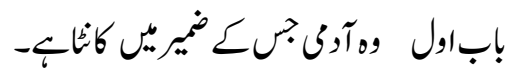

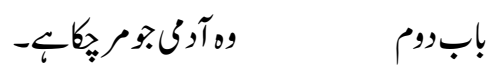
وه آرك.وزنزهـباب (بوم 


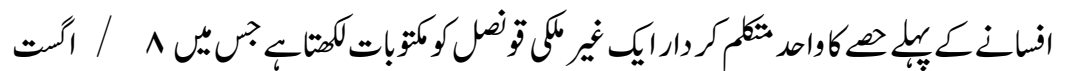

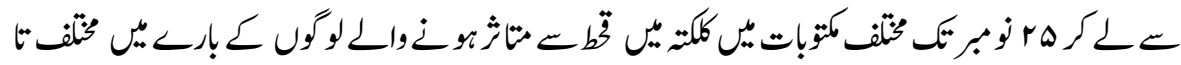

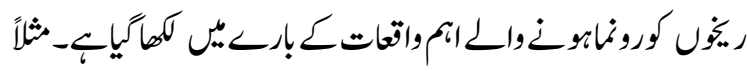

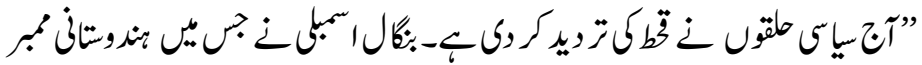

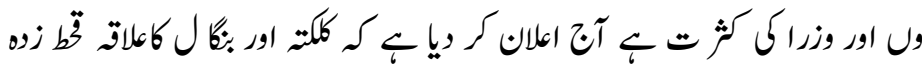

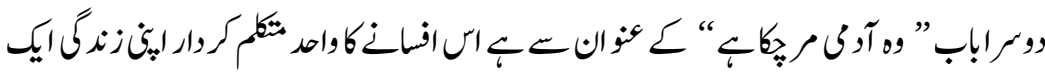

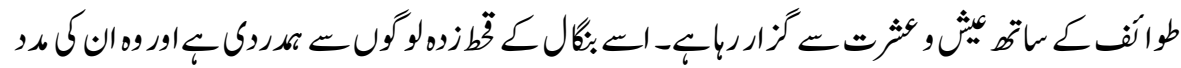

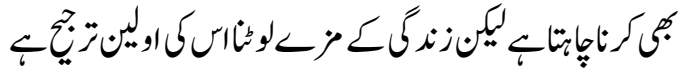

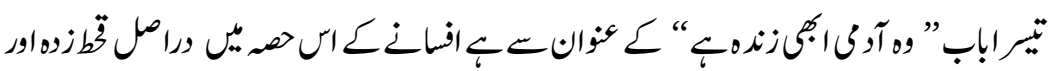

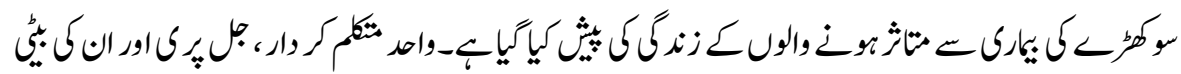

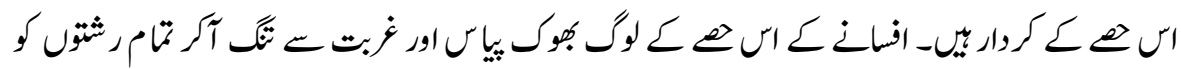

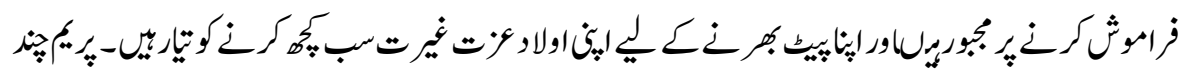

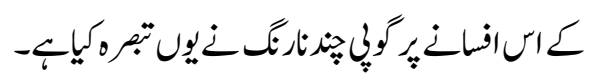

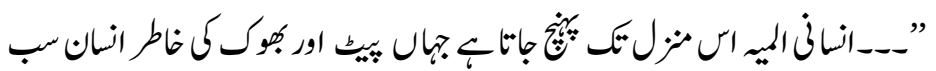

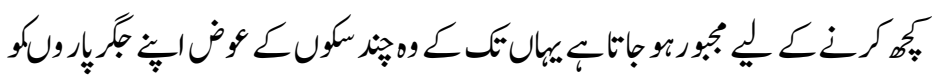

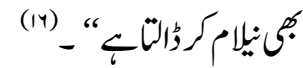

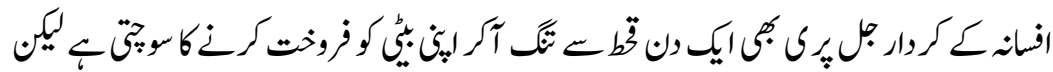

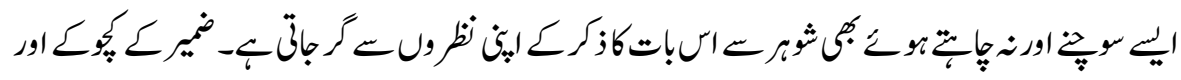

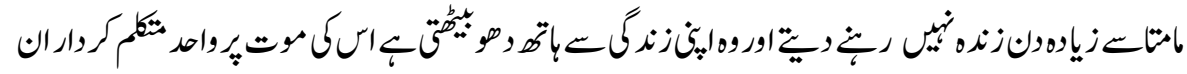

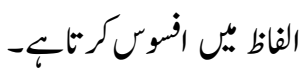

"_....

(1L) $-i^{4}$ 
ISSN (Print): 2709-9636 | ISSN(Online) : 2709-9644 | ISSN-L : 2709-9636

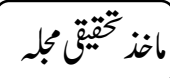

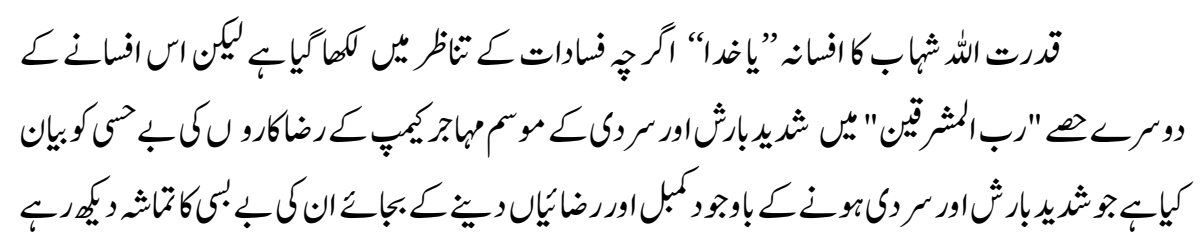

$-\vec{E}$

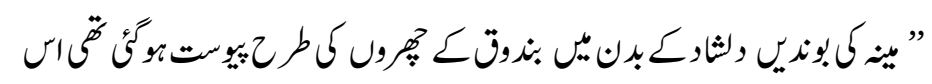

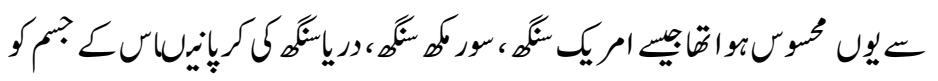

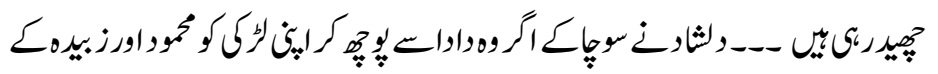

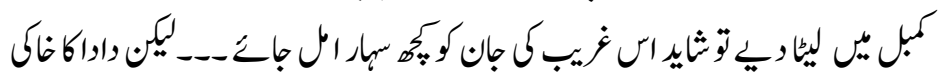

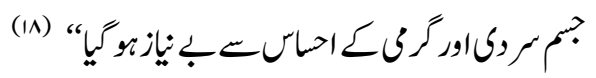

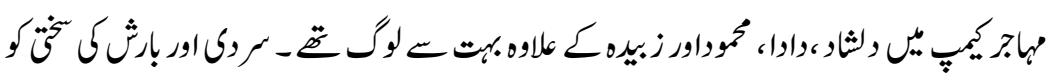

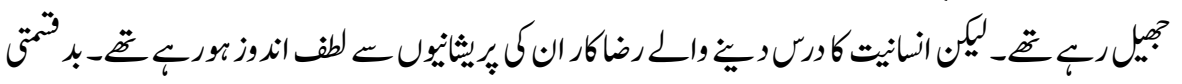

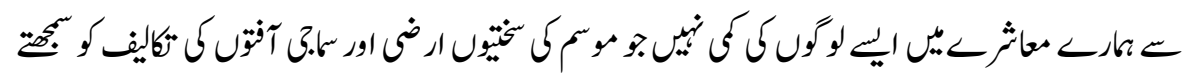

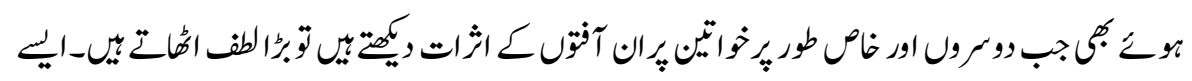

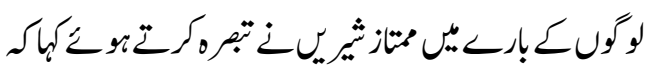

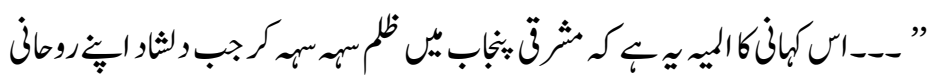

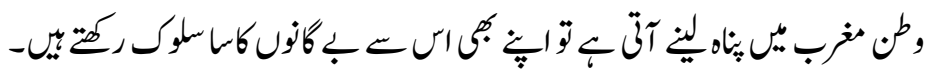

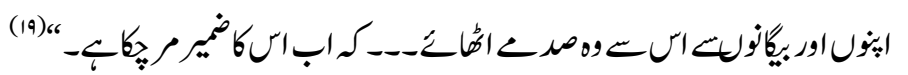

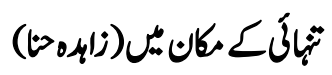

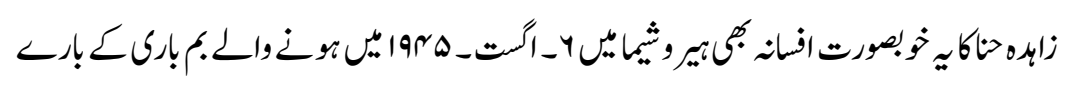

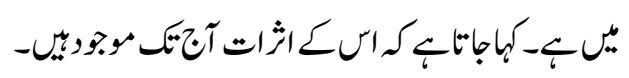

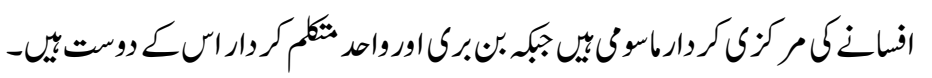

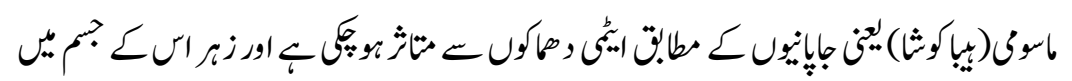

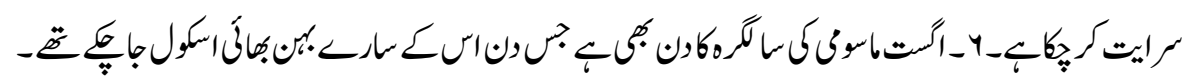

$1<1$ 


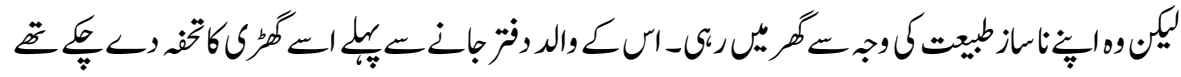

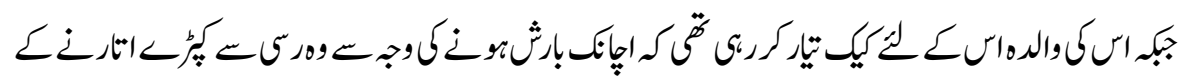

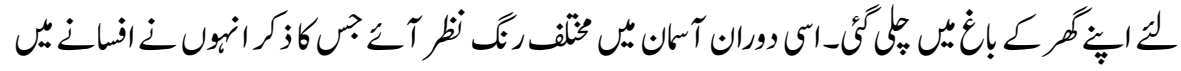

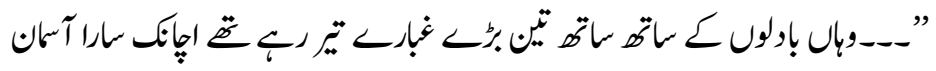

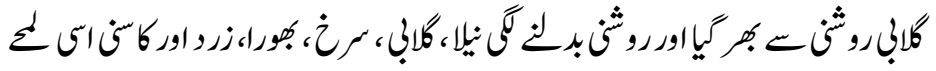

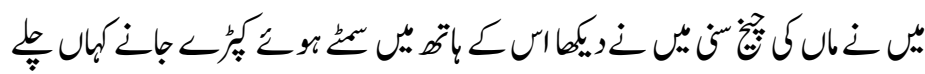
ك

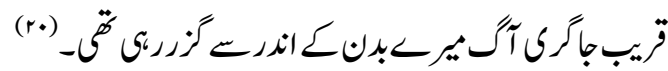

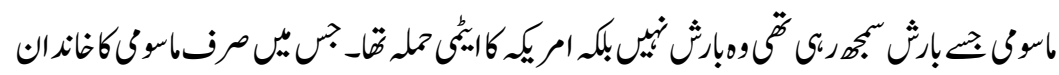

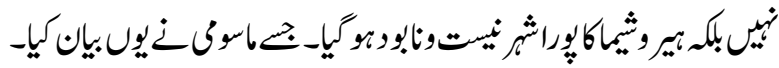

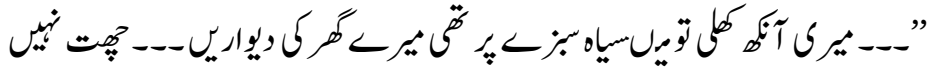

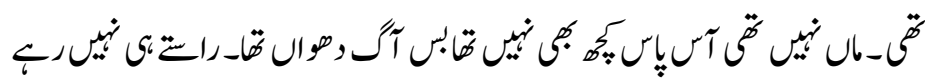

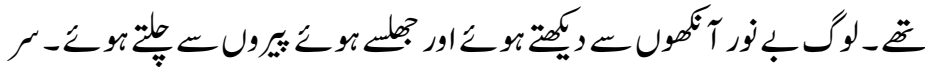

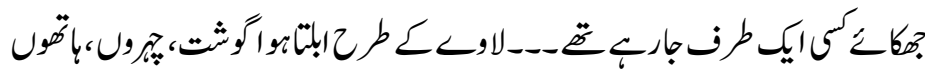

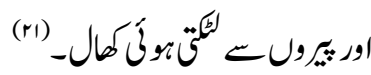

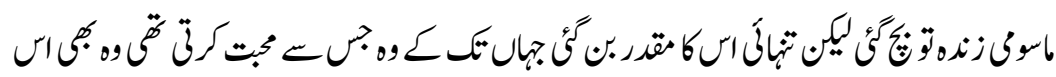

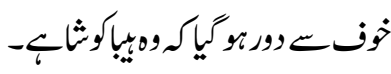
"خاف، "خواييول"

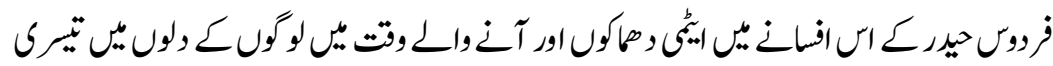

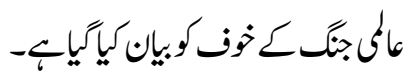

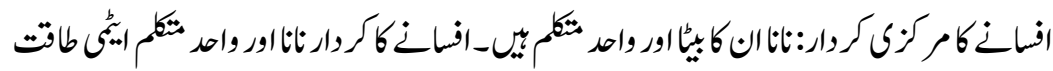




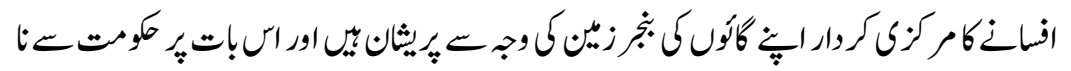

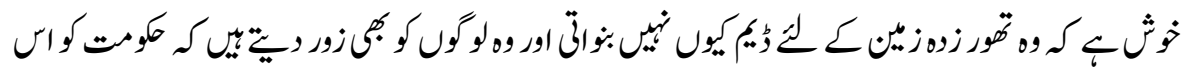

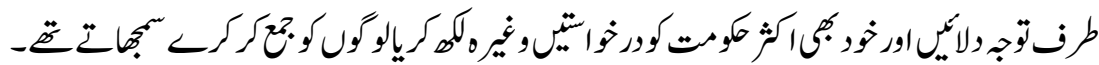

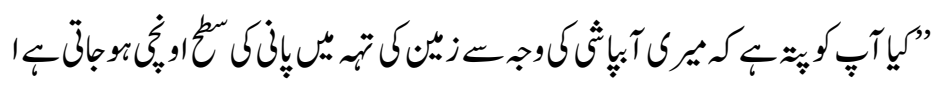

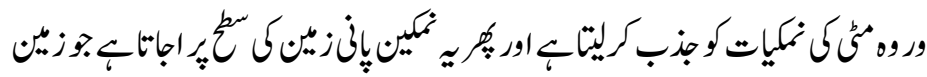
(rr)، -

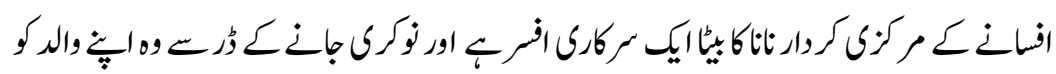

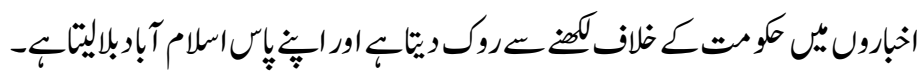

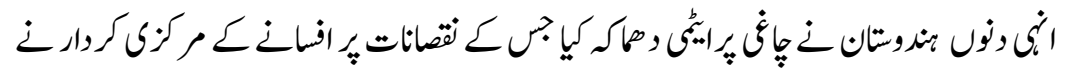

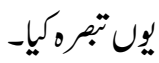

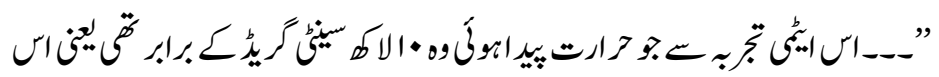

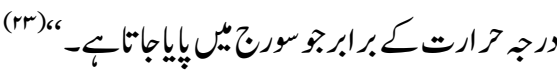

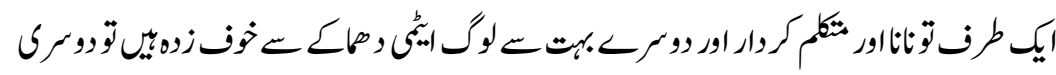

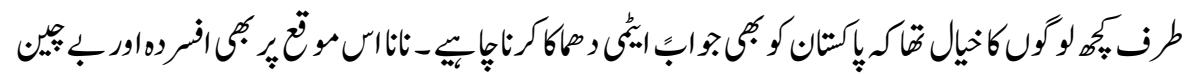

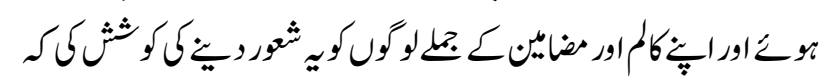

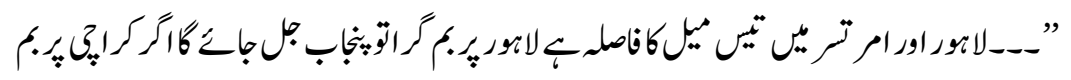

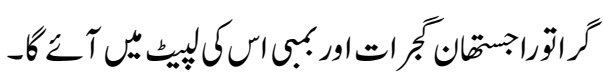

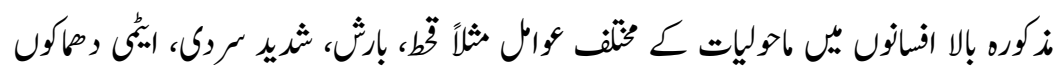

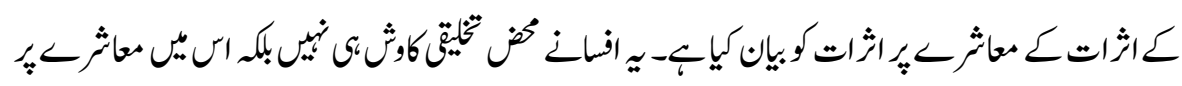

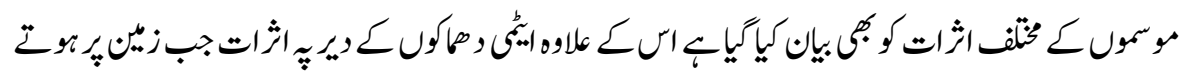

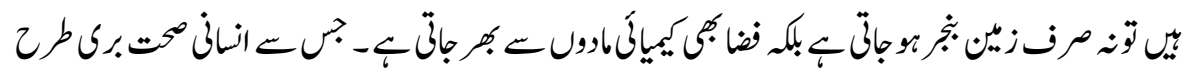
متاثرونَ 


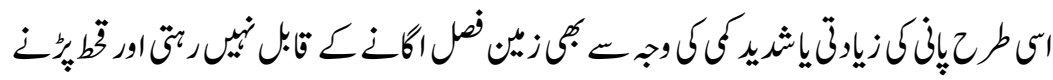

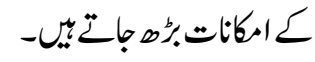

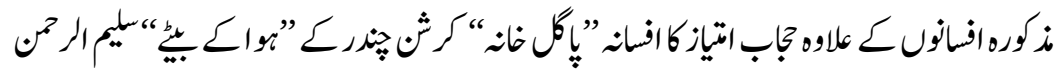

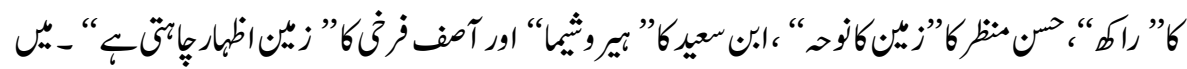

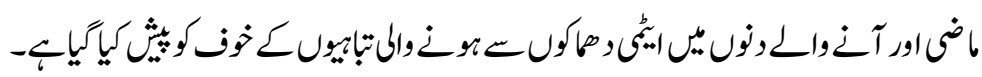

$$
\text { والرجات }
$$

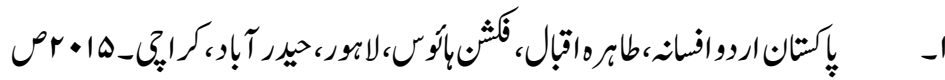

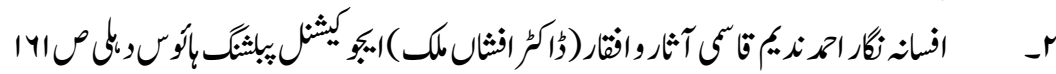

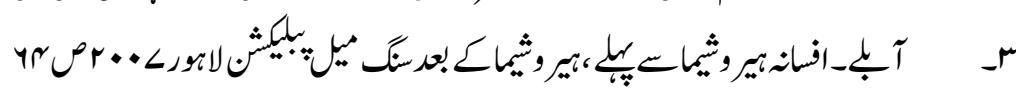
r

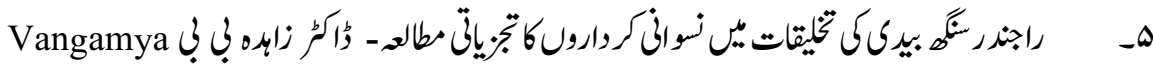

$$
\text { Book }
$$

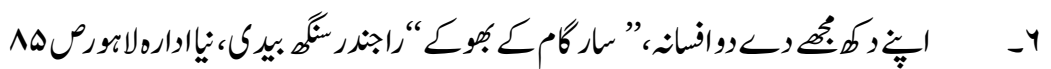

ك- - - -

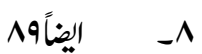

ب-

•ا- اليضأهو

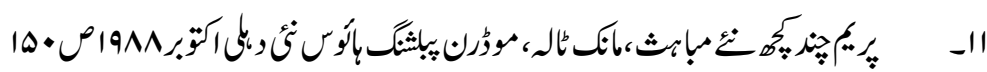

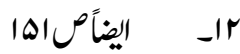

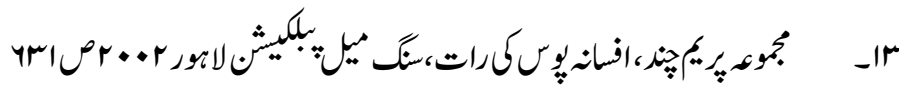

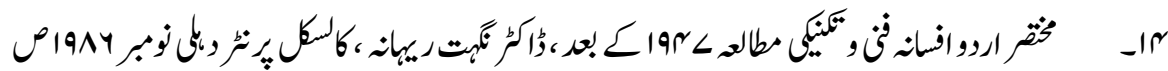

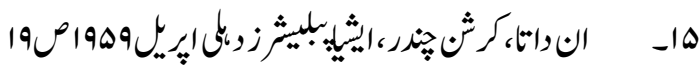




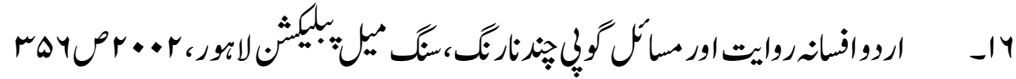

$$
\begin{aligned}
& \text { Lا- }
\end{aligned}
$$

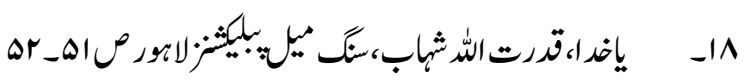

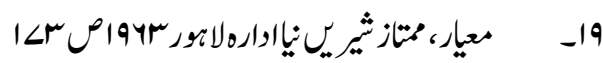

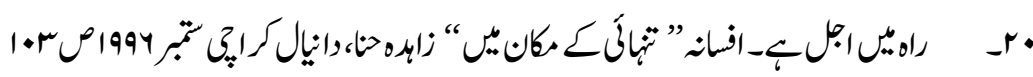

$$
\begin{aligned}
& \text { ا إيضاً }
\end{aligned}
$$

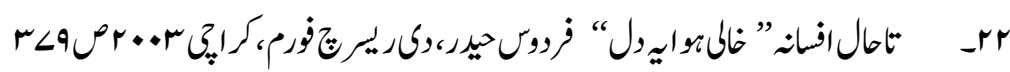

r r

r 